\title{
Barbara Stollberg-Rilinger, Tim Neu, Christina Brauner (dir.), Alles nur symbolisch? Bilanz und Perspektiven der Erforschung symbolischer Kommunikation
}

Köln, Weimar, Wien : Böhlau (Symbolische Kommunikation in der Vormoderne. Studien zur Geschichte, Literatur und Kunst), 2013, 472 p., $59,90 €$.

Falk Bretschneider

\section{(2) OpenEdition}

Édition électronique

URL : http://journals.openedition.org/ifha/8360

DOI : 10.4000/ifha.8360

ISSN : 2198-8943

Éditeur

IFRA - Institut franco-allemand (sciences historiques et sociales)

Référence électronique

Falk Bretschneider, «Barbara Stollberg-Rilinger, Tim Neu, Christina Brauner (dir.), Alles nur symbolisch? Bilanz und Perspektiven der Erforschung symbolischer Kommunikation », Revue de I'IFHA [En ligne], Date de recension, mis en ligne le 01 janvier 2016, consulté le 22 septembre 2020. URL : http:// journals.openedition.org/ifha/8360 ; DOI : https://doi.org/10.4000/ifha.8360

Ce document a été généré automatiquement le 22 septembre 2020.

(C)IFHA 


\section{Barbara Stollberg-Rilinger, Tim Neu, Christina Brauner (dir.), Alles nur symbolisch? Bilanz und Perspektiven der Erforschung symbolischer Kommunikation}

Köln, Weimar, Wien : Böhlau (Symbolische Kommunikation in der Vormoderne. Studien zur Geschichte, Literatur und Kunst), 2013, 472 p., $59,90 €$.

\section{Falk Bretschneider}

Parmi les Sonderforschungsbereiche (SFB) financés par la Deutsche Forschungsgemeinschaft dans les universités allemandes, certains réussissent à s'imposer non seulement comme les porteurs et les marqueurs d'un courant innovateur particulier (ce qu'on attend d'eux, d'ailleurs) mais de surcroît comme les porte-drapeaux d'une réorientation de la recherche dans son ensemble. Cela fut le cas du désormais mythique SFB 177 « Histoire sociale de la bourgeoisie moderne » (1986-1997), fleuron de l'école d'histoire sociale de Bielefeld, et cela risque fort d'être aussi celui du SFB 496 «Communication symbolique et systèmes normatifs des sociétés du Moyen Âge jusqu'à la Révolution française ", en activité de 2000 à 2011 à l'université de Münster où, autour de chercheurs éminents comme Gerd Althoff et Barbara Stollberg-Rilinger, s'est développée une nouvelle approche d'histoire culturelle tournée non pas vers l'analyse des discours (en vogue dans l'historiographie allemande des années 1990) mais vers l'étude des formes symboliques et rituelles de l'agir social et donc vers un examen approfondi du (ou des) sens que les acteurs donnaient eux-mêmes à leur histoire (cf. à ce sujet le $\mathrm{n}^{\circ} 2$ de la revue Trivium, «Culture politique et communication symbolique», http:// trivium.revues.org/793). Le présent recueil publie les contributions d'un grand colloque de clôture, organisé au printemps 2011 pour «marquer symboliquement et 
rituellement »- comme le notent les éditeurs non sans un brin d'humour (p. 9) - la fin des travaux de cette importante structure collective de la recherche.

La forme du livre est par conséquent celle du bilan: dans une introduction riche et dense, B. Stollberg-Rilinger et T. Neu passent d'abord en revue les douze ans d'activité $\mathrm{du} \mathrm{SFB}$, les principaux traits et résultats du concept, mais aussi ses limites. En se démarquant d'interprétations plus anciennes qui n'avaient voulu voir dans les rituels et les symboles qu'une pochette vide, un extérieur "purement symbolique " derrière lequel se cacherait une réalité "vraie", ils insistent encore une fois sur la force créative des actes performatifs de communication symbolique qui contribuent sans cesse à produire et à reproduire la réalité sociale des acteurs, en fabriquant des structures et du sens (p. 15). Fortement inspiré par la théorie luhmanienne, le concept fait de la communication un mode central d'action qui sert d'intermédiaire entre les structures 'objectives' et l'agir individuel, en intégrant d'une part le donné du monde dans lequel les acteurs évoluent, et d'autre part la nécessité de continuellement refonder les structures et de les inscrire dans la durée par des actes communicatifs. L'un des défis du SFB a ainsi été de montrer que même les actions dont les historiens soulignent traditionnellement le caractère instrumental (par exemple des actes du monde politique, comme des élections, ou du monde administratif, comme des délibérations) revêtent, elles aussi, des dimensions symboliques fondamentales, par exemple la représentation de l'ordre politique ou la légitimation des résultats d'une procédure, ce qui amène les éditeurs à une conclusion bienvenue : « non pas 'tout n'est que symbolique', mais 'tout est aussi symbolique' » (p. 25).

Huit chapitres rassemblent ensuite vingt-trois contributions qui donnent à voir la pluralité des champs d'application du concept. Gerd Althoff, Steffen Patzold et Frank Rexroth décrivent les « règles du jeu » de la communication symbolique au Moyen Âge, alors que Peter Oestmann et Susanne Lepsius s'interrogent sur ses fonctions dans le monde du droit médiéval (commentaire de Joachim Rückert). Le cérémonial diplomatique et ses pratiques symboliques et rituelles sont au centre des analyses de Lucien Bély et de Christian Windler (commentaire de Heinz Schilling), tandis que JeanClément Martin et Christina Schröer réfléchissent aux dimensions symboliques de la violence à l'époque de la Révolution française (commentaire de Jürgen Martschukat). Les trois chapitres suivants approfondissent le rôle de la production artistique (ces propos sont enrichis par une trentaine d'illustrations à la fin de l'ouvrage) : Jürgen Heidrich et Katelijne Schiltz examinent les formes et les limites de la communication symbolique dans la sphère musicale (commentaire de Laurenz Lütteken), Joachim Poeschke et Wolfgang Brassat étudient à l'exemple de portraits princiers et de tapisseries les fonctions symboliques, représentatives et décoratives des arts plastiques (commentaire de Klaus Krüger), Jan-Dirk Müller et Doris Kolesch reprennent le thème du rapport entre "jeu » et réalité, en s'intéressant à des formes modernes d'une performativité théâtrale (carnaval, entrées festives, hommages, mais aussi promenades royales dans le parc de Versailles) qui était pourtant profondément inscrite dans le contexte pragmatique de l'exercice du pouvoir (commentaire de Cornelia Herberichs). Enfin, dans le dernier chapitre consacré aux dimensions symboliques des actions économiques, les auteurs - Werner Freitag et T. Neu (commentaire de Simon Teuscher) - soulignent à juste titre qu'elles sont encore trop rarement prises en compte par l'historiographie (y compris dans le cadre du SFB münstérien). 
Malheureusement, le livre ne reprend pas les commentaires qu'ont effectués, lors du colloque, quelques représentants d'autres Sonderforschungsbereiche plus ou moins proches des mêmes problématiques, et qui ont abordé les forces et les faiblesses du concept à la lumière d'autres approches (cf. le compte-rendu du colloque sur HSozKult, http://www.hsozkult.de/conferencereport/id/tagungsberichte-3740). De même, le lecteur pourrait être troublé de constater que les résultats de travaux se réclamant d'un concept explicitement interdisciplinaire ont été rangés selon les disciplines. Cette démarche obéit toutefois à une volonté expresse des éditeurs (p. 25), celle de démontrer également les limites de l'entreprise (si bien que, pour certaines disciplines, sa pertinence reste peut-être encore en partie à démontrer). Ce parti-pris - ainsi que le choix de conserver la forme orale de la plupart des contributions, ce qui permet de suivre le dialogue qui s'est établi d'une intervention à l'autre - font donc de ce recueil important bien plus qu'un simple acte symbolique et rituel "de comm'» (tel qu'on l'attend aujourd'hui de chaque grand projet de recherche qui se termine), mais plutôt un bilan très honnête d'un concept qui a déjà fait ses preuves mais continuera certainement encore longtemps à marquer et à féconder la recherche, pas seulement en histoire.

Vous trouverez la table des matières ici : http://d-nb.info/1029521573/04.

INDEX

Thèmes : Histoire de la culture

Index chronologique : Période moderne

\section{AUTEUR}

FALK BRETSCHNEIDER

EHESS Paris 